\title{
Recommended Vaccination During Pregnancy: A Short Commentary
}

\section{VINESHA PANDITA}

Vaccination is the process of stimulating a protective adaptive immune response against microbes by exposure to

non-pathogenic forms or microbial components. Live vaccines killed vaccines and pathogen derived purified macromolecules are the three types of vaccines. Maternal vaccination provide protection to both the mother and fetus from the morbidity of certain serious infectious diseases which can otherwise be prevented by vaccination. Influenza and Pertussis vaccines are routinely recommended during pregnancy. Vaccines can also result in adverse reactions both in the mother and child. This review focuses on guidelines and recommendations for vaccination during pregnancy.

KEYWORDS: Vaccination, Pregnancy, Immunization, Vaccine

\section{INTRODUCTION}

As serious infections can harm the baby during pregnancy or after delivery, vaccinations in pregnancy, are of great concern. Vaccine-preventable diseases cause significant morbidity and mortality among mothers, neonates, and young infants. Immunization of the pregnant mother provides important health benefits limited not only to her but also to the fetus.

Vaccines help to strengthen the human immune system that can fight off serious infectious diseases. Development of immunity after vaccination protects the mother as well as the keeps the child safe during early months of life until the baby gets his/her own vaccination. ${ }^{1}$ While vaccination recommendations have changed sharply over the last few years on one hand, many of us, including physicians, are no longer familiar with the problems and complications of bygone diseases on the other. These diseases no longer seem worrisome, concerns about possible side effects and unfounded fears are frequently widespread. It is very important however that doctors and patients are wellinformed about the importance of vaccinations prior to and especially during pregnancy ${ }^{2,3}$ since this concerns the protection of the mother, the fetus and the newborn.

Broadly, vaccines can be classified as live or attenuated vaccines, killed or inactivated vaccines and pathogen derived purified macromolecules. A few vaccines are recommended prior to pregnancy, some are recommended during pregnancy, a few may be administered during pregnancy, only if necessary and some are also absolutely contraindicated during pregnancy. It is important to check the vaccination status of women who are planning to become pregnant. Gynecologists play a key role in counselling patients regarding vaccinations since a gynecological consultation is often the only medical attention a young woman may seek. Ideally, women should be examined before a planned pregnancy and vaccinated if necessary.

Measles mumps and rubella vaccinations are of great importance in addition to hepatitis A and B, diphtheria and tetanus as well as vaccinations against human papilloma viruses. Although these three typical pediatric diseases are fortunately, rare nowadays, it is worth bearing in mind why they were so feared as well as the importance of protecting women and their unborn children against them. Rubella often goes unnoticed, but pregnant women have an increased risk of having an abortion, or the disease itself may lead to severe fetal malformations, especially of the heart, of the eye leading to blindness, of the ear resulting in deafness or of the brain with resultant intellectual disability. The risk is especially high in the first trimester and decreases from the 2oth week onwards. Measles infection in pregnancy carries a high risk of abortion, premature or stillbirths. Fetal malformations do not occur. In infants, measles often leads to complications as well as frequent and prolonged hospitalizations. Measles infections in pregnant women 
may lead to severe pneumonia or meningitis. Mumps in pregnancy may increase the risk of abortion. Women who have not had varicella in childhood and have not been vaccinated should be administered the varicella vaccine, since varicella gives rise to complications more frequently in adults and especially in pregnant women than in children. Infection in the first half of pregnancy may result in fetal malformations with severe damage to the skin, bones, eyes or brain. Peripartum varicella infection in the mother may result in neonatal varicella, which is associated with high neonatal mortality. Depending on the season, influenza vaccination may be worthwhile before a planned pregnancy. Additionally, vaccinations such as Yellow fever vaccination may be indicated depending on lifestyle. Pregnancy should be excluded while administering live vaccines.

Vaccination during pregnancy plays a very special role, protecting the mother and fetus on one hand and providing passive immunity to the new-born on the other. Studies have shown that pregnant women are more susceptible to certain infectious diseases, while others often follow a more severe course. Various mechanical and physiological changes occur during pregnancy, such as reduced lung volume, increased heart rate and immunological adaptation mechanisms. It is important to be aware that healthy pregnant women may develop an immune response in the same manner as non-pregnant women. ${ }^{4}$ Vaccination against pertussis is currently recommended for all pregnant women. While pregnant women are not at high risk of developing a severe pertussis infection, newborns and infants carry a risk of developing this infection and have an increased risk of complications. The infection is associated with high morbidity, especially in the first few months of life. The most common sources of infection are family members. The pertussis vaccine does not provide long-lasting protection. Adequate protection against pertussis is expected after two doses, that is, four months at the earliest. However, since many severe illnesses occur in the first two to three months of life, the mother should be vaccinated during pregnancy, regardless of her vaccination status. Maternal antibodies are formed after vaccination which cross the placenta to the fetus and thus protect the newborn from infection. ${ }^{5}$

According to current recommendations, pregnant women should be administered the pertussis vaccine in the second trimester, so that premature babies may benefit from the vaccination. Vaccination induces the development of an adequate level of antibodies in the child even at 36 weeks of pregnancy. ${ }^{6}$ The vaccine may be administered in the puerperium if the mother's last vaccination was more than ten years ago and was not repeated during pregnancy. Although the vaccine prevents the mother from being the source of infection, this vaccination cannot reliably protect the newborn in the vulnerable phase. Pertussis vaccination is currently not known to be associated with adverse effects, either on the course of pregnancy or on fetal development.

Changes in the immune system during pregnancy result in increased susceptibility to infections, although this is less frequently seen with influenza. The physiological, hemodynamic and respiratory changes in pregnancy, however, predispose to more severe outcomes. Lung capacity decreases, while heart rate and stroke volume increase with an increase in gestational age. Pregnant women are thus more susceptible to severe disease, which leads to more frequent hospitalizations and increased mortality. ${ }^{7}$ Influenza may cause serious respiratory complications in pregnant women, especially during the $2^{\text {nd }}$ and $3^{\text {rd }}$ trimesters as well as in the first three months after delivery. It is also believed that influenza may also lead to complications such as prematurity or intrauterine growth retardation. ${ }^{8}$

Infection is associated with higher mortality and a higher risk of complicated outcomes in the first six months of a child's life. ${ }^{9}$ Influenza vaccination during pregnancy reduces the risk of the disease in pregnant women, thereby significantly lowering the risk of complications and hospitalization. The fetus is also protected by vaccination. ${ }^{10}$ There are fewer premature births and fewer instances of growth retardation. ${ }^{11}$ Vaccination during pregnancy also reduces the risk of infection in infants in the first few months of life. ${ }^{12}$ According to current knowledge, influenza vaccination in pregnancy has no adverse effects on the course of pregnancy or development of the fetus. Side effects have not been described more frequently in pregnant women. The vaccination is clearly recommended by the World Health Organization.

Vaccinations before and during pregnancy are gaining in importance. A paradigm change has furthermore occurred. While vaccinations were avoided as far as possible during pregnancy, two vaccinations are recommended nowadays during pregnancy in keeping with current recommendations. Firstly, the pertussis vaccine, which provides passive protection for the newborn in the first few weeks of life and secondly, the influenza vaccine which actively protects the mother during pregnancy and lactation. The fetus and newborn are also protected against possible influenza. 
Only live vaccines are contraindicated in pregnancy. Accidental vaccinations are not considered a reason for termination of pregnancy. If vaccinations are not administered prior to or during pregnancy, they should be rescheduled postpartum in the puerperal period.

If vaccinations are administered during pregnancy, the benefits for mother and child should outweigh the risk of vaccination. ${ }^{3}$ This is clearly the case for both influenza and pertussis vaccinations. All but live vaccines may in principle be administered during pregnancy if indicated. If a trip is planned to a polioendemic region, vaccination with the inactivated parenteral polio vaccine or one of a combination vaccine against poliomyelitis may be administered. Pneumococcal or Hemophilus influenzae vaccinations may also be indicated under certain circumstances. Live vaccines are contraindicated in pregnancy since they theoretically carry a potential risk of fetal infection. Although countless publications show that accidental vaccination against rubella in the first trimester does not lead to rubella embryopathy, MMR and other live vaccines are contraindicated in pregnancy. Reliable contraception is recommended for four weeks after administration of live vaccines. ${ }^{3}$

\section{CONCLUSION}

An important strategy to improve pregnancy outcomes is vaccination during pregnancy. Live attenuated viral and bacterial vaccines are generally contraindicated during pregnancy because of the risks to the fetus. Vaccines like drugs can cause adverse reactions and intervention during pregnancy can affect both mother and child, therefore, vaccines with relative and absolute contraindications should be avoided during pregnancy. Pertussis and influenza vaccines are indicated during pregnancy. Pregnant mothers should be vaccinated only if the benefits to the mother and the fetus should outweigh the risks. Awareness regarding both benefits of maternal vaccination and its safety concerns needs to be generated. Proper immunization programs should be organized by the governmental health bodies to involve each and every pregnant mother so that health of two individuals is boosted using one vaccination.

\section{REFERENCES}

1. Verma R, Khanna P, Dhankar M. Vaccination during pregnancy: Today's need in India. Hum Vaccin Immunother. 2015;12:668-70.

2. Mueller Y, Eberhardt CS, Diana A, Martinez De Tejada B. Recommendations for the vaccination of pregnant women: a booster injection. Rev Med Suisse. 2016;12:1708-12.

3. Munoz FM, Jamieson DJ. Maternal Immunization. Obstet Gynecol. 2019;133:739-53.

4. Chu HY, Englund JA. Maternal immunization. Birth Defects Res 2017;109:379-86.

5. Englund JA. The influence of maternal immunization on infant immune responses. J Comp Pathol. 2007;137:16-1.

6. Abraham C, Pichichero M, Eisenberg J, Singh S. Third-trimester maternal vaccination against pertussis and pertussis antibody concentrations. Obstet Gynecol. 2018;131:364-9.

7. Kourtis AP, Read JS, Jamieson DJ. Pregnancy and infection. N Engl J Med. 2014;371:1077.

8. Naresh A, Fisher BM, Hoppe KK, et al. A multicenter cohort study of pregnancy outcomes among women with laboratory confirmed $\mathrm{H}_{1} \mathrm{~N}_{1}$ influenza. J Perinatol. 2013;33:939-43.

9. Zaman K, Roy E, Arifeen SE, et al. Effectiveness of maternal influenza immunization in mothers and infants. N Engl J Med. 2008;359:1555-64.

10. Poehling KA, Szilagyi PG, Staat MA, et al. Impact of maternal immunization on influenza hospitalizations in infants. Am J Obstet Gynecol. 2011;204:141-8.

11. Nunes MC, Aqil AR, Omer SB, Madhi SA. The effects of influenza vaccination during pregnancy on birth outcomes: A systematic review and meta-analysis. Am J Perinatol. 2016;33:1104-14.

12. Madhi SA, Nunes MC, Cutland CL. Influenza vaccination of pregnant women and protection of their infants. N Engl J Med. 2014;371:918-31. 
Source of support: Nil, Conflict of interest: None declared

Cite this article as:

Pandita V. Recommended Vaccination During Pregnancy: A Short Commentary. Int

Healthc Res J. 2020;4(3):52-55. https://doi.org/10.26440/IHRJ/0403.06173

\section{AUTHOR AFFILIATIONS:}

1. MDS (Public Health Dentistry), Consultant Private Practitioner and Ex-Senior Lecturer, Swami Devi Dyal Hospital and Dental College,

Barwala, Panchkula, India

Contact corresponding author at: drvineshapandita[at]gmail[dot]com 